\title{
Regional consultative meeting on primary health care for universal health coverage ${ }^{1}$
}

Citation: Regional consultative meeting on primary health care for universal health coverage. East Mediterr Health J. 2019;25(12):928-929 https://doi. $\operatorname{org} / 10.26719 / 2019.25 .12 .928$

Copyright @ World Health Organization (WHO) 2019. Some rights reserved. This work is available under the CC BY-NC-SA 3.0 IGO license (https:// creativecommons.org/licenses/by-nc-sa/3.o/igo).

\section{Introduction}

A regional consultative meeting on primary health care (PHC) for universal health coverage (UHC) took place on 30 July-1 August 2019 in Cairo, Egypt (1). The meeting saw the formal launch of the Primary Health Care Measurement and Improvement (PHCMI) initiative (2), which follows from the launch of the Primary Health Care Performance Initiative (PHCPI) announced by Dr Ahmed Al-Mandhari, WHO Regional Director for the Eastern Mediterranean Region, at the celebration of World Health Day on 5 April 2019, in Cairo, Egypt (3). The initiative aims to support countries to fulfil the commitments made in the Astana Declaration on PHC (4).

The three-day meeting was attended by ministry of health focal persons for PHC and health information from 20 Eastern Mediterranean Region (EMR) countries, as well as PHCMI initiative partners, representatives from the Bill \& Melinda Gates Foundation and the World Organization of Family Doctors (WONCA), as well as WHO country office health systems focal points.

The objectives of the meeting were to:

- share the current status of PHC implementation in the EMR as related to the recommendations of the Astana Declaration and the PHCMI initiative;

- disseminate the findings of the PHC assessment conducted in three pilot countries (Egypt, Jordan and Pakistan) as part of the Measurement Phase of the PHCMI initiative;

- establish a mechanism for integrating PHC services in the Region with the goal of attaining health for all; and

- explore the health system requirements - in terms of governance, workforce, financing, technologies, infrastructure and information - needed to ensure successful implementation of the PHCMI initiative to enhance UHC in the Region.

Results from the initiative will be driven by three primary goals: 1 ) building regional and national capacity and awareness for an enhanced evidence-based approach to PHC improvement; 2) institutionalizing PHC measurement in existing health system performance assessments; and 3) improving PHC performance/ scaling-up of family practice to help countries accelerate progress towards the achievement of UHC.

\section{Summary of discussions}

A series of presentations were delivered during the meeting regarding the health systems contribution to PHC. This included presentations on: financing frameworks and tools for effective implementation of PHC for UHC; the importance of investment in the health workforce for PHC; effective and quality essential medicines and technologies; viewing hospital roles and operations through an integrated and people-centred lens; the challenges in quality and safety in health systems, with recommendations for improvement; improving health service delivery by implementing robust health information systems; and building resilient health systems as a prerequisite to achieving UHC.

It was noted that challenges to the health workforce remain, including maldistribution, brain drain and increased mobility. Benefits' packages, in the context of a country, also need to take into account the scope of practice of the health workforce. The move to familybased practice PHC is another challenge for the Region, since training family physicians will take years and will require additional transitional measures. Participants agreed that bridging programmes to encourage general practitioners to become family physicians are needed, rather than relying on specialist family medicine programmes and financial incentives. However, this will not be sufficient; strengthening the health workforce to improve PHC will also require reliable and accurate data.

\section{Recommendations}

\section{To Member States}

- Providing feedback on the Implementation Manual.

- Appointing a focal point for the remaining countries without one (Saudi Arabia and Kuwait).

- For the pilot countries (Egypt, Jordan and Pakistan), beginning the Improvement Phase and commencing the action plans developed in the meeting. Egypt and Pakistan have been recommended for partner collaboration for PHC implementation, which will increase technical (and potentially increase

\footnotetext{
${ }^{1}$ This summary is extracted from the report on the Regional consultative meeting on primary health care for universal health coverage, Cairo, Egypt, 30 July-1 August 2019 (http://applications.emro.who.int/docs/IC_Meet_Rep_2019_PHC_175_EN.pdf?ua=1).
} 
financial) support for PHC strengthening in the two countries.

- For Egypt and Jordan, beginning the Measurement Phase in close collaboration with WHO.

- Continuing close coordination with WHO and partner organizations towards a second regional workshop.

\section{To WHO}

- Finalizing PHC quality indicators and disseminate to Member States.

- Continuing close coordination between the different levels of WHO and with countries and partner organizations towards a second regional workshop 3-5 December 2019.

\section{References}

1. World Health Organization Regional Office for the Eastern Mediterranean (WHO/EMRO). Regional consultative meeting on primary health care for universal health coverage, Cairo, Egypt, 30 July-1 August 2019 (http://applications.emro.who.int/docs/ IC_Meet_Rep_2019_PHC_175_EN.pdf?ua=1).

2. World Health Organization. Assessing, measuring, improving PHC. Geneva: World Health Organization; 2019 (https://www.who. int/activities/assessing-measuring-improving-phc).

3. World Health Organization Regional Office for the Eastern Mediterranean (WHO/EMRO). World Health Day 2019: WHO and partners join hands in solidarity for universal health coverage. Cairo: WHO/EMRO; 2019 (http://www.emro.who.int/media/news/ world-health-day-2019-who-and-partners-join-hands-in-solidarity-for-universal-health-coverage.html).

4. World Health Organization (WHO) and the United Nations Children's Fund (UNICEF). Declaration of Astana. Geneva: WHO/ UNICEF; 2018 (https://www.who.int/docs/default-source/primary-health/declaration/gcphc-declaration.pdf). 